Originally published as:

Leendertz, F.H., Deckers, M., Schempp, W., Lankester, F., Boesch, C., Mugisha, L., Dolan, A., Gatherer, D., McGeoch, D.J., Ehlers, B.

Novel cytomegaloviruses in free-ranging and captive great apes: Phylogenetic evidence for bidirectional horizontal transmission

(2009) Journal of General Virology, 90 (10), pp. 2386-2394.

DOI: 10.1099/vir.0.011866-0

This is an author manuscript that has been accepted for publication in Microbiology, copyright Society for General Microbiology, but has not been copy-edited, formatted or proofed. Cite this article as appearing in Microbiology. This version of the manuscript may not be duplicated or reproduced, other than for personal use or within the rule of 'Fair Use of Copyrighted Materials' (section 17, Title 17, US Code), without permission from the copyright owner, Society for General Microbiology. The Society for General Microbiology disclaims any responsibility or liability for errors or omissions in this version of the manuscript or in any version derived from it by any other parties. The final copy-edited, published article, which is the version of record, can be found at http://mic.sgmjournals.org, and is freely available without a subscription 12 months after publication. 


\title{
Novel cytomegaloviruses in free-ranging and captive great apes: phylogenetic evidence for bidirectional horizontal transmission
}

\author{
Fabian H. Leendertz, ${ }^{1}$ Merlin Deckers, ${ }^{2}$ Werner Schempp, ${ }^{3}$ Felix Lankester, ${ }^{4}$ Christophe Boesch, ${ }^{5}$ \\ Lawrence Mugisha, ${ }^{6}$ Aidan Dolan, ${ }^{7}$ Derek Gatherer, ${ }^{7}$ Duncan J. McGeoch ${ }^{7}$ and Bernhard Ehlers ${ }^{2}$ \\ ${ }^{1}$ Research Group Emerging Zoonoses, Robert Koch-Institut, D-13353 Berlin, Germany \\ ${ }^{2}$ Research Group Molecular Genetics and Epidemiology of Herpesviruses, Robert Koch-Institut, \\ D-13353 Berlin, Germany \\ ${ }^{3}$ Institute for Human Genetics and Anthropology, University of Freiburg, D-79106 Freiburg, \\ Germany \\ ${ }^{4}$ Limbe Wildlife Centre, PO Box 878, Limbe, Cameroon \\ ${ }^{5}$ Max Planck Institute for Evolutionary Anthropology, Department of Primatology, Deutscher Platz 6, \\ D-04103 Leipzig, Germany \\ ${ }^{6}$ Chimpanzee Sanctuary and Wildlife Conservation Trust (CSWCT), PO Box 884, Entebbe, Uganda \\ ${ }^{7}$ Medical Research Council Virology Unit, Institute of Virology, University of Glasgow, Church Street, \\ Glasgow G11 5JR, UK
}

Correspondence: Bernhard Ehlers ehlersb@rki.de

\begin{abstract}
Wild great apes often suffer from diseases of unknown aetiology. This is among the causes of population declines. Because human cytomegalovirus (HCMV) is an important pathogen, especially in immunocompromised individuals, a search for cytomegaloviruses (CMVs) in deceased wild and captive chimpanzees, gorillas and orang-utans was performed. By using a degenerate PCR targeting four conserved genes (UL54-UL57), several distinct, previously unrecognized CMVs were found for each species. Sequences of up to $9 \mathrm{~kb}$ were determined for ten novel CMVs, located in the UL54UL57 block. A phylogenetic tree was inferred for the ten novel CMVs, the previously characterized chimpanzee CMV, HCMV strains and Old World and New World monkey CMVs. The primate CMVs fell into four clades, containing New World monkey, Old World monkey, orang-utan and human CMVs, respectively, plus two clades that each contained both chimpanzee and gorilla isolates (termed CG1 and CG2). The tree loci of the first four clades mirrored those for their respective hosts in the primate tree, suggesting that these CMV lineages arose through cospeciation with host lineages. The CG1 and CG2 loci corresponded to those of the gorilla and chimpanzee hosts, respectively. This was interpreted as indicating that CG1 and CG2 represented CMV lineages that had arisen cospeciationally with the gorilla and chimpanzee lineages, respectively, with subsequent transfer within each clade between the host genera. Divergence dates were estimated and found to be consistent with overall cospeciational development of major primate CMV lineages. However, CMV transmission between chimpanzees and gorillas in both directions has also occurred.
\end{abstract}

\section{Introduction}

Cytomegaloviruses (CMVs; family Herpesviridae, subfamily Betaherpesvirinae) are ubiquitous and mostly inapparent agents that exist in many mammals, including humans and other primates. Human cytomegalovirus (HCMV) can induce serious diseases in individuals lacking fully competent immune functions, such as transplant recipients, AIDS patients and newborns (reviewed by Ehlers, 2008; Mocarski et al., 2006). The determinants, duration and severity of HCMV disease are not well resolved. Host factors such as the strength of cellular and humoral immune responses are of importance. In addition, the occurrence of different genotypic variants may influence HCMV virulence and hence the outcome of disease. However, the linkage of certain HCMV variants to strain-specific properties, such as tissue tropism, and to defined patterns of pathogenesis is - although studied by numerous laboratories - still a matter of controversial debate (reviewed by Pignatelli et al., 2004).

In the closest relatives of humans, the great apes, only one CMV has been described unequivocally, the chimpanzee cytomegalovirus (CCMV; Panine herpesvirus 2; formerly Pongine herpesvirus 4). The 
pathogenesis of CCMV infection is essentially unknown. The genome of CCMV has been sequenced completely and compared with the HCMV genome, revealing general collinearity and also regions of substantial divergence (Davison et al., 2003).

Infectious disease has joined habitat loss and hunting as a threat to the survival of the remaining wild populations of great apes (Leendertz et al., 2006), but relatively little is known about the causative agents. We investigated an unusually high number of sudden deaths observed between 1999 and 2006 in three communities of wild chimpanzees (Pan troglodytes verus) in the Taï National Park, Ivory Coast. A number of bacterial and viral pathogens were identified as likely causes, including novel strains of a Bacillus anthracis-related bacterium, as well as viruses and bacteria causing respiratory diseases (Leendertz et al., 2004; Köndgen et al., 2008). In order to investigate possible cofactors for these diseases, we searched for other pathogens,

including herpesviruses, by using a highly efficient universal PCR system (Ehlers et al., 1999; Chmielewicz et al., 2003; reviewed by Ehlers, 2008). This work revealed the presence of a previously unknown chimpanzee CMV, significantly different from the characterized CCMV and related more closely to HCMV. This finding prompted us to investigate in depth the CMV lineages and their evolution in great apes.

\section{Methods}

Sample collection and processing, PCR methods and sequence analysis.

Over a time range of 10 years, blood, tissue and faeces samples were collected from live or deceased West African chimpanzees (Pan troglodytes verus) in Taï National Park, Ivory Coast (Leendertz et al., 2006), and in Guinea; East African chimpanzees (Pan troglodytes schweinfurthii) in Uganda (housed at the Ngamba Island Chimpanzee Sanctuary) and in the Democratic Republic of the Congo; Western lowland gorillas (Gorilla gorilla gorilla) in Cameroon; and orang-utans (Pongo pygmaeus pygmaeus) in Malaysia. Samples were also collected from live or deceased individuals, including an Eastern lowland gorilla (Gorilla gorilla graueri), in several zoological gardens in Germany, Italy and South Africa. Details of the samples that yielded sequence data are available on request. DNA was prepared with a QIAamp tissue kit according to the manufacturer's instructions (Qiagen). For universal detection of herpesviruses, pan-herpes DPOL-PCR for amplification of 160-181 bp (excluding primer-binding sites) of the DNA polymerase (DPOL) gene (UL54 in HCMV) was carried out as described previously (Chmielewicz et al., 2003). For detection of betaherpesviruses only, sequences of the DPOL gene, the glycoprotein B (gB) gene [open reading frame (ORF) UL55], the UL56 gene and the major DNAbinding protein (MDBP) gene (ORF UL57) of as-yetunknown CMVs were amplified with six degenerate, deoxyinosinesubstituted primer sets (see Supplementary Table S1, available in JGV Online), based on the respective genes of HCMV (strain AD169; GenBank accession no. NC_001347). The primer sites were located in regions conserved among the betaherpesviruses. The primers were only minimally degenerate in order to avoid amplification of viruses related to human herpesviruses 6 and 7, as well as alpha- and gammaherpesviruses. PCR was carried out at an annealing temperature of $46 u C$ under the conditions used in pan-herpes DPOL-PCR. To amplify different sequences from the same, multi-infected primate sample of viruses closer to CCMV or toHCMV, specific (non-degenerate) primers (not listed), targeting conserved regions of UL54 or UL55, were deduced from either the CCMV genome or the HCMV genome. Amplifications were done in a nested format. Long-distance nested PCR was performed with the TaKaRa-Ex PCR system (TaKaRa Bio) according to the manufacturer's instructions, using virus-specific primers (not listed).

PCR product purification and direct sequencing with dye-terminator chemistry, as well as nucleotide sequence analysis and amino acid sequence prediction, were performed as described previously (Goltz et al., 2002).

Inference of phylogenetic trees and dating of nodes. Sets of inferred amino acid sequences were aligned by using MAFFT (Katoh et al., 2002). Alignments were cleaned before being used for phylogenetic analysis by removal of regions that were considered not to be justifiably alignable and of loci with a gapping character in any sequence. The JTT substitution table for amino acid residues was used in all tree inferences and modelling (Jones et al., 1992). Phylogenetic trees were derived by Bayesian analysis using Monte Carlo Markov chains (BMCMC) with MrBayes v. 3.1 (Ronquist \& Huelsenbeck, 2003), as described previously (Ehlers et al., 2008). Assignments of dates to nodes on CMV phylogenetic trees were made by two methods. In the first, molecular clock trees with topology based on the BMCMC-derived trees were calculated by using CODEML (PAML package v. 4; Yang, 
2007), with a discrete gamma distribution of five classes of substitution rate across sites. The tree node representing the common ancestor of Old World and New World primate CMVs was used as the single calibration point, assigned the estimated date for divergence of Old World and New World primates, and dates for other nodes in the tree were obtained from branch lengths by proportion. The second method was by BMCMC with the program BEAST v. 1.4, which combines tree inference and node dating with a relaxed clock model (Drummond et al., 2006; Drummond \& Rambaut, 2007). The same calibration point was used, specified as a normal distribution. A gamma+invariant distribution of rates over sites and a relaxed, uncorrelated, log-normal molecular clock specification were employed. The tree prior was specified as a Yule process, appropriate for trees representing speciation events. The prior for the mean rate of evolution over the entire tree was alternatively specified as uniform- or gamma-distributed; these priors gave closely similar estimated dates. BMCMC chains were run by using BEAST's auto-optimization function until effective sample sizes for all parameters were .300 .

Provisional nomenclature, abbreviations and nucleotide sequence accession numbers for the novel herpesviruses.

The viruses from which the novel sequences originated were named after the host species name and the herpesvirus genus to which the virus was tentatively assigned (e.g. Pan troglodytes cytomegalovirus, PtroCMV). The genotypic variants of PtroCMV that were related

more closely to CCMV than to HCMV (genogroup 1) were named PtroCMV-1.1 and PtroCMV-1.2. Those related more closely to HCMV (genogroup 2) were named PtroCMV-2.1 and PtroCMV-2.2,

etc. The variants of gorilla CMV (GgorCMV-1 and -2), orang-utan CMV (PpygCMV-1 and -2) and Old World monkeys (MfasCMV-1; MsphCMV-1) were named accordingly. All novel viruses are listed with their abbreviations and GenBank accession numbers in Table 2, together with those of previously sequenced viruses that were analysed for comparison.

\section{Results and Discussion}

\section{De novo detection of novel CMVs}

To elucidate the diversity of CMV in great apes, we analysed 335 samples from West African and East African chimpanzees (n5168), Western lowland and Eastern lowland gorillas (n5107) and orang-utans (n560). The majority of the individuals were from the wild.

In initial tests for the presence of herpesviruses, pan-herpes DPOL-PCR was carried out on all samples and reaction products were sequenced. Those samples yielding CMVlike sequences were analysed further. Samples in which herpesviruses other than betaherpesviruses were detected with pan-herpes DPOL-PCR were reanalysed with the nested degenerate primer sets P54, P55-1 and P552 (Supplementary Table S1), which were restricted to targeting parts of the DPOL and gB genes, respectively, of HCMV and non-human primate CMVs only. In total, 36 samples were CMV-positive in these assays. For each host species, samples that had the most diverged sequences and high copy numbers, as deduced from the appearance of strong amplification products after PCR, were then

selected. These were subjected to further testing with the primer sets P56, P57-1 and P57-2 (Supplementary Table S1), targeting ORFs UL56 and UL57, respectively, of HCMV and non-human primate CMVs.

We next attempted to connect the partial sequences originating from two to four genes (UL54-UL57). This was complicated by the fact that most animals were apparently infected with more than one CMV. We dealt with this difficulty by subjecting all partial sequences amplified from a given CMV gene to phylogenetic tree reconstruction. We then compared the four resulting trees (for UL54, UL55, UL56 and UL57) and tentatively defined sequences that had comparable positions in the trees as originating from the same virus genome. These were then connected by long-distance PCR. Based on this approach, we were able to generate contiguous sequences of 2.1-9.0 kb (UL55-UL56, UL55-UL57 or UL54-UL57) originating from ten novel CMVs of three great ape species, namely West African chimpanzees, Western lowland gorillas and orang-utans (Table 1). Of the ten CMVs detected, eight were found in primates that had lived in the wild. Nine CMVs were found in more than one specimen, nine in more than one animal and nine in animals from different locations. The majority of the tested primate individuals had mixed infections. All CMVs were provisionally named and are listed with their host species and GenBank accession numbers in Tables 1 and 2.

Among the great apes, chimpanzees are the only hosts for which CMVs have been described previously (Alcendor \& 
Hayward, 2008; Eberle \& Hilliard, 1989; McCarthy \& Tosolini, 1975; Swinkels et al., 1984). A CMV-like infection in a captive lowland gorilla has been reported previously, but solely on the basis of histopathological findings (Tsuchiya et al., 1970). Genetic evidence has been published for the existence in gorillas and orang-utans of herpes simplex viruslike alphaherpesviruses and Epstein-Barr virus-like gammaherpesviruses, but not betaherpesviruses (Ehlers et al., 2003; Luebcke et al., 2006). Therefore, the novel CMV sequences from Western lowland gorillas and orang-utans presented here indicate for the first time and unequivocally the existence of CMVs in these great ape species.

\section{Comparison of sequences and phylogenetic analysis}

Pairwise comparisons of DNA and amino acid sequences from the newly detected great ape CMVs plus CCMV and HCMV showed that they fell into four major clusters: one of HCMV strains, one of the two PpygCMVs and two that each contained CMVs from both chimpanzees and gorillas (Table 3).

Formal phylogenetic analysis was then applied to examine relationships further. Analysis of $\mathrm{gB}$ amino acid sequences proved most productive and was carried out in greatest detail, based on sequences from the ten novel great ape CMVs, CCMV and four strains of HCMV, plus eight sequences from Old World monkey (OWM) CMVs, two from New World monkey (NWM) CMVs, and three nonprimate viruses, as detailed in Tables 1 and 2. OWM CMVs represented were: rhesus CMV (RhCMV; Macacine herpesvirus 3); two CMV variants from Colobus guereza (CgueCMV-1.1 and -1.2) (Prepens et al., 2007); CMVs from Macaca fascicularis (MfasCMV-1), Mandrillus sphinx (MsphCMV-1) and Papio anubis (BaCMV); and two additional simian CMVs, Cercopithecine herpesvirus 5 (CeHV-5) and simian CMV strain Colburn. MfasCMV-1 and MsphCMV-1 had been detected in the course of a search for primate gammaherpesviruses (Ehlers et al., 2003), and their UL54-UL57 sequences (8.1 and 8.2 $\mathrm{kb}$, respectively) were amplified in the same way as described above for the great ape viruses. CeHV5 and Colburn CMV sequences were extracted from complete genome sequences (GenBank accession numbers in Table 2). NWM CMVs represented were SsciCMV-1 from Saimiri sciureus and AtriCMV-1 from Aotus trivirgatus (from complete genome sequences; GenBank accession numbers in Table 2). Sequences for murine CMV (MCMV), rat CMV (RCMV) and tupaiid herpesvirus 1 (TuHV-1) were included to provide an outgroup to the primate CMVs, based on previous analysis (McGeoch et al., 2006). In total, 28 partial gB sequences gave an alignment of 420 aa after removal of highly diverged loci and positions with a gapping character in any sequence; this was evaluated phylogenetically by BMCMC. A single phylogenetic tree was obtained, as shown in Fig. 1(a), with maximum posterior probability for all branching features except for three closely spaced terminal branch points within the two clades of chimpanzee plus gorilla CMVs. The primate CMV sequences were divided cleanly into six major clades, labelled in Fig. 1(a) as NWM CMVs, OWM CMVs, PpygCMVs (two PpygCMV strains), CG1 CMVs (containing genogroup 1 chimpanzee and gorilla CMVs), CG2 CMVs (containing genogroup 2 chimpanzee and gorilla CMVs) and HCMV. A longer alignment of 629 aa, constructed with 22 complete and near-complete gB sequences, gave a closely comparable tree (not shown). A concatenated alignment for gB (UL55) plus UL56 sequences was constructed, containing 23 sequences and 1127 aa in length, and evaluated by BMCMC. The resulting tree, shown in Fig. 1(b), had maximum posterior probability for all branching features (except within the closely spaced HCMV clade) and its branching pattern was completely consistent with that in Fig. $1(a)$.

The phylogenetic relationships among the six major clades of primate CMVs revealed in these trees are equivalent in most respects to those among the primate hosts, as outlined in the cladograms in Fig. 2. Specifically, the CMV clades labelled in Figs 1 and 2(a) as NWM CMVs, OWM CMVs, PpygCMVs and HCMV correspond exactly in their branching relationships to the NWM, OWM, Pongo and Homo clades, respectively, in the host cladogram (Fig. 2b). However, the CG1 clade in the locus corresponding to the host gorilla clade contains both chimpanzee and gorilla viruses, as does the CG2 clade in the locus corresponding to the host chimpanzee clade. The simplest, and most compelling, resolution of all these features is that the primate CMV tree does represent, in its large-scale features, a coevolutionary development with the host lineages; that CG1 and CG2 are the cospeciational clades for gorilla and chimpanzee CMVs, respectively; and that transmission across host genera, i.e. from gorillas to chimpanzees, has occurred within CG1, and vice versa within CG2. In each of CG1 and CG2, a single cross-species transmission event would account for the observed branch structure. This view of overall mode in the descent of primate CMVs is compatible with the wider context in the subfamily Betaherpesvirinae, where apparent cospeciational features extending beyond the primate CMVs have been pointed out (McGeoch et al., 2006).

As enunciated above, this hypothesis of cospeciational development of primate CMV lineages was based on branching pattern. We next evaluated the equivalence of the virus and host trees by 
comparisons of relative branch proportions. We regarded the divergence of New World and Old World primate CMVs as being the most convincing cospeciational feature, with no plausible alternative scenarios visible, and we therefore took this tree node ( $A$ in Fig. 2a) to correspond to the palaeontologically dated divergence of New World and Old World primate lineages, then inferred dates for other nodes in the virus tree and compared these with dates for the host tree. As a general caveat, it should be noted that estimates of primate divergence dates are neither exact nor finalized: published figures continue to change (most often to deeper dates) and also have large associated confidence intervals. We based our analyses on the work of Steiper \& Young (2006), using their estimate of 42.9 MA (millions of years before present) for the split of New World and Old World primates. Dates for nodes in the CMV tree were then derived in two ways. The simplest approach is to construct, by a maximum-likelihood method, a tree corresponding to that in Fig. 1(a), but with a global molecular clock imposed, and then to estimate dates of nodes by

proportion relative to the reference node. The second method comprises a detailed probabilistic modelling process that combines tree inference and node dating carried through by BMCMC with a relaxed clock model, using the program BEAST (Drummond et al., 2006). It turned out that with the primate CMV datasets that we used, the two approaches gave closely similar results, so only the BEAST datings are reported here. Results are presented in Table 4 for the major nodes in the CMV tree, for both the 420 aa UL55 and 1127 aa UL55+UL56 alignments, and are compared with the corresponding dates for the host tree. Overall, the CMV estimates of date ranges sit comfortably with the host dates in Table 4, although it would of course be desirable to have smaller confidence intervals in all cases, and we regard the dating exercises as supportive of the hypotheses of general cospeciation and of interspecies transfer of chimpanzee and gorilla CMVs.

A possible alternative interpretation is that separation of the CMV lineages represented by CG1 and CG2 could predate speciation of the host lineages leading to chimpanzee and gorilla, and that within each of CG1 and CG2, the split into chimpanzee CMV strains and gorilla CMV strains could then correspond to cospeciation with the host lineages. This scenario has the compelling disadvantage that, within the larger picture of primate CMV cospeciational development presented by the clades of NWM CMVs, OWM CMVs and PpygCMVs, it would require rates of change in the CG1 and CG2 lineages to be much slower than in these other lineages, and it does not provide a rationalization for the location of the HCMV clade.

This paper is not aimed primarily at the NWM and OWM CMVs, but we register some points regarding details within these clades (Fig. 1). In the NWM CMV clade, we estimated that AtriCMV-1 and SsciCMV-1 diverged around $20 \mathrm{MA}$, and this date is similar to that estimated for divergence of the host genera Aotus and Saimiri (Schneider, 2000). In the OWM CMV clade, there are four relatively deep lineages, for CMVs of macaque, colobus, mandrill plus baboon, and cercopithecus species, whose branching patterns differ in part from the hosts' phylogeny, specifically in that the host tree has colobus diverging first from the other lineages (Raaum et al., 2005). There is thus some complexity of relationships yet to be uncovered in the OWM clade. Last, the Colburn CMV strain was isolated from human brain in a case of encephalopathy (Huang et al., 1978), but clearly belongs to the OWM CMV clade (Fig. 1).

In summary, the following was concluded: primate CMVs evolved with their hosts in a cospeciational mode, but horizontal transmission of CMVs has apparently occurred between chimpanzees and gorillas in both directions.

CMVs generally have a host range that is restricted to cells from the species they infect (Mocarski et al., 2006). However, this specificity is not absolute, as MCMV replicates in human and simian cells and simian CMV (strain Colburn) in human cells (Lafemina \& Hayward,

1988). Efficient replication of rhesus CMV and baboon CMV in cultured human endothelial and epithelial cells was reported (Michaels et al., 1997; Lilja \& Shenk, 2008). Isolates of chimpanzee CMV were grown on human diploid embryonic lung cells (Swinkels et al., 1984) and on human fibroblasts and brain cells (Wroblewska et al., 1979). Vice versa, HCMV was reported to infect primary chimpanzee fibroblasts (Perot et al., 1992). These examples, together with the data presented here, may indicate that primate CMVs are not exclusively species-specific and have the potential to transmit horizontally to hosts related closely to their natural host. This may be of importance with regard to the frequent handling of primates, their meat and organs in countries with populations of great apes. The most cogent example in this context is, of course, the emergence of human immunodeficiency viruses from origins in various simian immunodeficiency viruses (Hahn et al., 2000). 
Specifically, our findings raise the question of whether additional CMVs exist in humans that have been transmitted zoonotically from great apes. Such infections might have been unrecognized to date if they occurred only locally in Africa, were not detected in routine diagnostic PCR assays or were detected but not recognized as aberrant human CMV. The novel CMV sequences reported here will aid in designing primers for the universal and sensitive detection of primate CMV in humans.

HCMV is a facultative pathogen that causes disease in adults, particularly when their immune system is compromised, for example in AIDS patients and transplant recipients. In these clinical situations, the occurrence of multiple HCMV variants has been observed and linked to severity and progression of CMV disease (Coaquette et al.,

2004). In line with this, non-human primates suffering from bacterial and viral diseases may encounter disease exacerbation by reactivation of their own CMVs or by becoming infected exogenously. The observed simultaneous detection of several PtroCMV variants in diseased chimpanzee individuals may thus indicate a general property of primate CMV pathogenesis.

\section{Acknowledgements}

For excellent technical assistance we thank Sonja Liebmann, Nezlisah Yasmum, Judit Küchler and Claudia Hedemann. For work in Ivory Coast, we thank the Ivorian authorities for long-term support, especially the Ministry of the Environment and Forests as well as the Ministry of Research, the directorship of the Taï National Park, and the Swiss Research Centre in Abidjan. For work in Cameroon, we thank the Ministry of Forests and Fauna and the staff of the Limbe Wildlife Centre, especially Dr Kiyang John Anyam. For samples from Uganda, we thank the CSWCT for permission to collect samples. For providing faeces samples from wild orang-utans, we thank Marc Ancrenaz from the Kinabatangan Wildlife Sanctuary.

\section{References}

Alcendor, D. J. \& Hayward, G. S. (2008). Cytomegaloviruses: simian cytomegaloviruses. In Encyclopedia of Virology, 3rd edn, pp. 634-642.

Edited by W. J. Mahy \& M. H. V. Van Regenmortel. Amsterdam: Elsevier. Chmielewicz, B., Goltz, M., Lahrmann, K. H. \& Ehlers, B. (2003). Approaching virus safety in xenotransplantation: a search for unrecognized herpesviruses in pigs. Xenotransplantation 10, 349-356.

Coaquette, A., Bourgeois, A., Dirand, C., Varin, A., Chen, W. \& Herbein, G. (2004). Mixed cytomegalovirus glycoprotein B genotypes in immunocompromised patients. Clin Infect Dis 39, 155161.

Davison, A. J., Dolan, A., Akter, P., Addison, C., Dargan, D. J., Alcendor, D. J., McGeoch, D. J. \& Hayward, G. S. (2003). The human cytomegalovirus genome revisited: comparison with the chimpanzee cytomegalovirus genome. J Gen Virol 84, 17-28.

Drummond, A. J. \& Rambaut, A. (2007). BEAST: Bayesian evolutionary analysis by sampling trees. BMC Evol Biol 7, 214.

Drummond, A. J., Ho, S. Y., Phillips, M. J. \& Rambaut, A. (2006). Relaxed phylogenetics and dating with confidence. PLoS Biol 4, e88.

Eberle, R. \& Hilliard, J. K. (1989). Serological evidence for variation in the incidence of herpesvirus infections in different species of apes. J Clin Microbiol 27, 1357-1366.

Ehlers, B. (2008). Discovery of herpesviruses. In Encyclopedia of Virology, 3rd edn. Edited by W. J. Mahy \& M. H. V. Van Regenmortel. Amsterdam: Elsevier. Ehlers, B., Borchers, K., Grund, C., Frölich, K., Ludwig, H. \& Buhk, H.-J. (1999). Detection of new DNA polymerase genes of known and potentially novel herpesviruses by PCR with degenerate and deoxyinosine-substituted primers. Virus Genes 18, 211-220.

Ehlers, B., Ochs, A., Leendertz, F., Goltz, M., Boesch, C. \& Mätz-Rensing, K. (2003). Novel simian homologues of Epstein-Barr virus. J Virol 77, 10695-10699.

Ehlers, B., Dural, G., Yasmum, N., Lembo, T., de Thoisy, B., Ryser-Degiorgis, M. P., Ulrich, R. G. \& McGeoch, D. J. (2008). Novel mammalian herpesviruses and lineages within the Gammaherpesvirinae: cospectiation and interspecies transfer. J Virol 82, 3509-3516.

Goltz, M., Ericsson, T., Huang, C., Patience, C., Sachs, D. H. \& Ehlers, B. (2002). Sequence analysis of the genome of porcine lymphotropic herpesvirus 1 and gene expression during posttransplant lymphoproliferative disease of pigs. Virology 294, 383-393. 
Hahn, B. H., Shaw, G. M., De Cock, K. M. \& Sharp, P. M. (2000). AIDS as a zoonosis: scientific and public health implications. Science 287, 607-614.

Huang, E. S., Kilpatrick, B., Lakeman, A. \& Alford, C. A. (1978). Genetic analysis of a cytomegalovirus-like agent isolated from human brain. J Virol 26, 718-723.

Jones, D. T., Taylor, W. R. \& Thornton, J. M. (1992). The rapid generation of mutation data matrices from protein sequences. Comput Appl Biosci 8, 275-282.

Katoh, K., Misawa, K., Kuma, K. \& Miyata, T. (2002). MAFFT: a novel method for rapid multiple sequence alignment based on fast Fourier transform. Nucleic Acids Res 30, 3059-3066.

Köndgen, S., Kühl, H., N'Goran, P. K., Walsh, P. D., Schenk, S., Ernst, N., Biek, R., Formenty, P., Mätz-Rensing, K. \& other authors (2008). Pandemic human viruses cause decline of endangered great apes. Curr Biol 18, 260-264.

Lafemina, R. L. \& Hayward, G. S. (1988). Differences in cell-typespecific blocks to immediate early gene expression and DNA replication of human, simian and murine cytomegalovirus. J Gen Virol 69, 355-374.

Leendertz, F. H., Ellerbrok, H., Boesch, C., Couacy-Hymann, E., Mätz-Rensing, K., Hakenbeck, R., Bergmann, C., Abaza, P., Junglen, S. \& other authors (2004). Anthrax kills wild chimpanzees in a tropical rainforest. Nature 430, 451-452.

Leendertz, F. H., Pauli, G., Ellerbrok, H., Maetz-Rensing, K., Boardman, W., Jensen, S. A., Junglen, S. \& Boesch, C. (2006). Pathogens as drivers of population declines: the importance of systematic monitoring in great apes and other threatened mammals. Biol Conserv 131, 325-337.

Lilja, A. E. \& Shenk, T. (2008). Efficient replication of rhesus cytomegalovirus variants in multiple rhesus and human cell types. Proc Natl Acad Sci U S A 105, 19950-19955.

Luebcke, E., Dubovi, E., Black, D., Ohsawa, K. \& Eberle, R. (2006). Isolation and characterization of a chimpanzee alphaherpesvirus. J Gen Virol 87, 11-19.

McCarthy, K. \& Tosolini, F. A. (1975). A review of primate herpes viruses. Proc R Soc Med 68, 145150. McGeoch, D. J., Rixon, F. J. \& Davison, A. J. (2006). Topics in herpesvirus genomics and evolution. Virus Res 117, 90-104.

Michaels, M. G., Alcendor, D. J., St George, K., Rinaldo, C. R., Jr, Ehrlich, G. D., Becich, M. J. \& Hayward, G. S. (1997). Distinguishing baboon cytomegalovirus from human cytomegalovirus: importance for xenotransplantation. J Infect Dis 176, 1476-1483.

Mocarski, E. S., Jr, Shenk, T. \& Pass, R. F. (2006). Cytomegaloviruses. In Fields' Virology, 5th edn, pp. 2701-2772. Edited by D. M. Knipe \& P. M. Howley. Philadelphia, PA: Lippincott Williams \& Wilkins.

Perot, K., Walker, C. M. \& Spaete, R. R. (1992). Primary chimpanzee skin fibroblast cells are fully permissive for human cytomegalovirus replication. J Gen Virol 73, 3281-3284.

Pignatelli, S., Dal Monte, P., Rossini, G. \& Landini, M. P. (2004). Genetic polymorphisms among human cytomegalovirus (HCMV) wild-type strains. Rev Med Virol 14, 383-410.

Prepens, S., Kreuzer, K.-A., Leendertz, F., Nitsche, A. \& Ehlers, B. (2007). Discovery of herpesviruses in multi-infected primates using locked nucleic acids (LNA) and a bigenic PCR approach. Virol J 4, 84.

Raaum, R. L., Sterner, K. N., Noviello, C. M., Stewart, C.-B. \& Disotell, T. R. (2005). Catarrhine primate divergence dates estimated from complete mitochondrial genomes: concordance with fossil and nuclear DNA evidence. J Hum Evol 48, 237-257.

Ronquist, F. \& Huelsenbeck, J. P. (2003). MrBayes 3: Bayesian inference under mixed methods. Bioinformatics 19, 1572-1574.

Schneider, H. (2000). The current status of the new world monkey phylogeny. An Acad Bras Cienc 72, 165-172.

Steiper, M. E. \& Young, N. M. (2006). Primate molecular divergence dates. Mol Phylogenet Evol 41, 384-394.

Swinkels, B. W., Geelen, J. L., Wertheim-van Dillen, P., van Es, A. A. \& van der Noordaa, J. (1984). Initial characterization of four cytomegalovirus strains isolated from chimpanzees. 1984. Arch Virol 82, 125-128.

Tsuchiya, Y., Isshiki, O. \& Yamada, H. (1970). Generalized cytomegalovirus infection in a gorilla. Jpn J Med Sci Biol 23, 71-73.

Wroblewska, Z., Gilden, D., Devlin, M., Huang, E. S., Rorke, L. B., Hamada, T., Furukawa, T., Cummins, L., Kalter, S. \& Koprowski, H. (1979). Cytomegalovirus isolation from a chimpanzee with acute demyelinating disease after inoculation of multiple sclerosis brain cells. Infect Immun 25, 10081015.

Yang, Z. (2007). PAML 4: phylogenetic analysis by maximum likelihood. Mol Biol Evol 24, 1586-1591. 
Table 1 Primate species, novel CMVs and their sequences

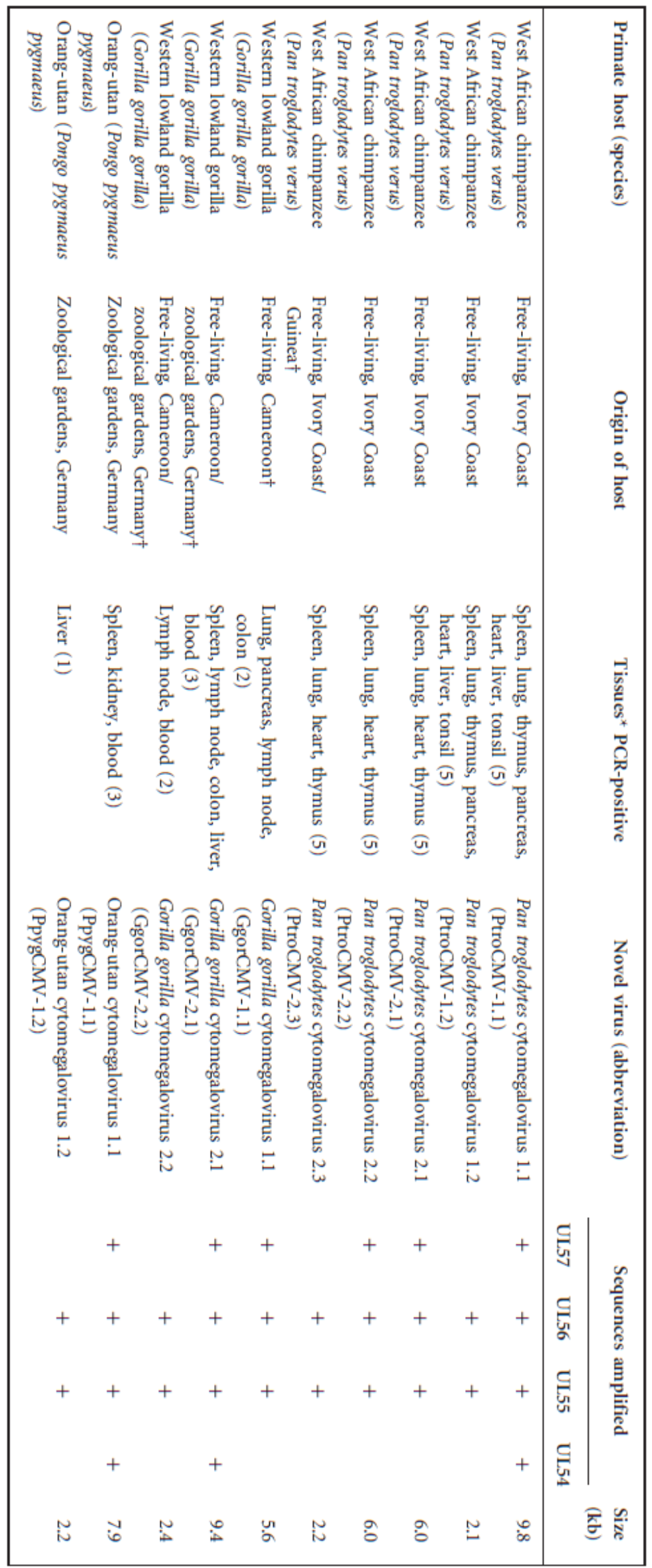

* Number of virus-positive primate individuals in parentheses.

† Wild-born but housed in a wildlife sanctuary. 
Table 2 Viruses, abbreviations and GenBank accession numbers

\begin{tabular}{|c|c|c|}
\hline Virus name & Abbreviation & GenBank accession no. \\
\hline \multicolumn{3}{|l|}{ Viruses from this study } \\
\hline \multicolumn{3}{|l|}{ UL54-UL57 sequences } \\
\hline Pan troglodytes cytomegalovirus 1.1 & PtroCMV-1.1 & F538485 \\
\hline Pan troglodytes cytomegalovirus 1.2 & PtroCMV-1.2 & FJ538486 \\
\hline Pan troglodytes cytomegalovirus 2.1 & PtroCMV-2.1 & FJ538487 \\
\hline Pan troglodytes cytomegalovirus 2.2 & PtroCMV-2.2 & FJ538488 \\
\hline Pan troglodytes cytomegalovirus 2.3 & PtroCMV-2.3 & F5538489 \\
\hline Gorilla gorilla cytomegalovirus 2.1 & GgorCMV-2.1 & FJ538490 \\
\hline Gorilla gorilla cytomegalovirus 2.2 & GgorCMV-2.2 & FJ538491 \\
\hline Gorilla gorilla cytomegalovirus 1.1 & GgorCMV-1.1 & FJ538492 \\
\hline Pongo pygmaeus cytomegalovirus 1.1 & PpygCMV-1.1 & AY129396 \\
\hline Pongo pygmaeus cytomegalovirus 1.2 & PpygCMV-1.2 & FJ538493 \\
\hline Macaca fascicularis cytomegalovirus 1 & MfasCMV-1 & AY728171 \\
\hline Mandrillus sphinx cytomegalovirus 1 & MsphCMV-1 & AY129399 \\
\hline \multicolumn{3}{|l|}{ Published viruses } \\
\hline \multicolumn{3}{|l|}{ Complete genomes } \\
\hline \multicolumn{3}{|l|}{ Human cytomegalovirus (Human herpesvirus 5) } \\
\hline Strain Merlin & HCMV Merlin & NC_006273 \\
\hline Strain Toledo & HCMV Toledo & $\mathrm{AC} 146905$ \\
\hline Strain Towne & HCMV Towne & AY315197 \\
\hline Strain AD169 & HCMV AD169 & NC_001347 \\
\hline Chimpanzee cytomegalovirus (Panine herpesvirus 2) & CCMV & NC_003521 \\
\hline Rhesus cytomegalovirus (Cercopithecine herpesvirus 8 ) & $\mathrm{RhCMV}$ & NC_006150 \\
\hline Cercopithecus aethiops cytomegalovirus (Cercopithecine herpesvirus 5) & CeHV-5 & FJ483968 \\
\hline Simian cytomegalovirus, strain Colburn & Colburn CMV & FJ483969 \\
\hline Saimiri sciureus cytomegalovirus 1 & SsciCMV-1 & FJ483967 \\
\hline Aotus trivirgatus cytomegalovirus 1 & AtriCMV-1 & FJ483970 \\
\hline Murine cytomegalovirus & MCMV, MuHV-1 & NC_001664 \\
\hline Rat cytomegalovirus & RCMV, MuHV-2 & NC_002512 \\
\hline Tupaiid herpesvirus 1 & TuHV-1 & NC_002794 \\
\hline \multicolumn{3}{|l|}{ UL54-UL57 sequences } \\
\hline Colobus guereza cytomegalovirus 1.1 & CgueCMV-1.1 & AY129397 \\
\hline Colobus guereza cytomegalovirus 1.2 & CgueCMV-1.2 & EU118147 \\
\hline Baboon cytomegalovirus & $\mathrm{BaCMV}$ & AF324835 \\
\hline
\end{tabular}

Table 3 Relationships of human and great ape CMVs

\begin{tabular}{|c|c|c|c|c|c|c|c|}
\hline \multirow[t]{3}{*}{ Virus } & \multicolumn{6}{|c|}{ Amino acid identity (\%) } & \multirow[t]{3}{*}{ Group } \\
\hline & \multicolumn{2}{|c|}{ UL55 } & \multicolumn{2}{|c|}{ UL56 } & \multicolumn{2}{|c|}{ UL54 } & \\
\hline & $\mathrm{HCMV}^{*}$ & CCMV & $\mathrm{HCMV}^{*}$ & CCMV & $\mathrm{HCMV}^{*}$ & CCMV & \\
\hline HCMV† & $93-95$ & $76-77$ & $99-100$ & 86 & 100 & 70 & 1 \\
\hline PtroCMV-2.1 & 81 & 75 & 91 & 86 & & & 2 \\
\hline PtroCMV-2.2 & 82 & 76 & & & & & 2 \\
\hline PtroCMV-2.3 & 81 & 75 & & & & & 2 \\
\hline GgorCMV-2.1 & 81 & 77 & 91 & 86 & 88 & 69 & 2 \\
\hline GgorCMV-2.2 & 82 & 76 & & & & & 2 \\
\hline PtroCMV-1.1 & 77 & 95 & 86 & 100 & 70 & 99 & 3 \\
\hline PtroCMV-1.2 & 76 & 97 & & & & & 3 \\
\hline GgorCMV-1.1 & 76 & 88 & 86 & 91 & & & 3 \\
\hline PpygCMV-1.1 & 75 & 75 & 77 & 78 & 75 & 62 & 4 \\
\hline PpygCMV-1.2 & 75 & 74 & & & & & 4 \\
\hline
\end{tabular}

\section{* Strain Merlin.}

† Strains Toledo and AD169. 
Table 4 Estimates of dates for nodes in the primate CMV tree Dates are given as MA (millions of years before present) and ranges are 95\% confidence limits.

\begin{tabular}{|lccc|}
\hline $\begin{array}{c}\text { Node }^{*} \\
\text { UL55 (gB) } 420 \text { aa alignment } \\
(28 \text { sequences) }\end{array}$ & $\begin{array}{c}\text { UL55 + UL56 1357 aa } \\
\text { alignment (21 sequences) }\end{array}$ & Host dates $\dagger$ \\
\hline A & 42.9 input $\ddagger$ & 42.9 input $\neq$ & $42.9(37.3-52.4) \ddagger$ \\
B & $31.2(24.0-38.1)$ & $27.7(21.3-34.0)$ & $30.5(26.9-36.4)$ \\
C & $22.9(16.6-29.8)$ & $21.3(15.6-27.1)$ & $18.3(16.3-26.8)$ \\
D & $15.4(10.6-20.6)$ & $11.3(7.8-15.0)$ & $8.6(7.7-9.2)$ \\
E & $9.9(6.4-13.9)$ & $6.9(4.6-9.7)$ & $6.6(6.0-7.0)$ \\
\hline
\end{tabular}

*Node labels refer to Fig. 2.

† Data of Steiper \& Young (2006).

‡ Input calibration date of 42.9 MA 
(a)

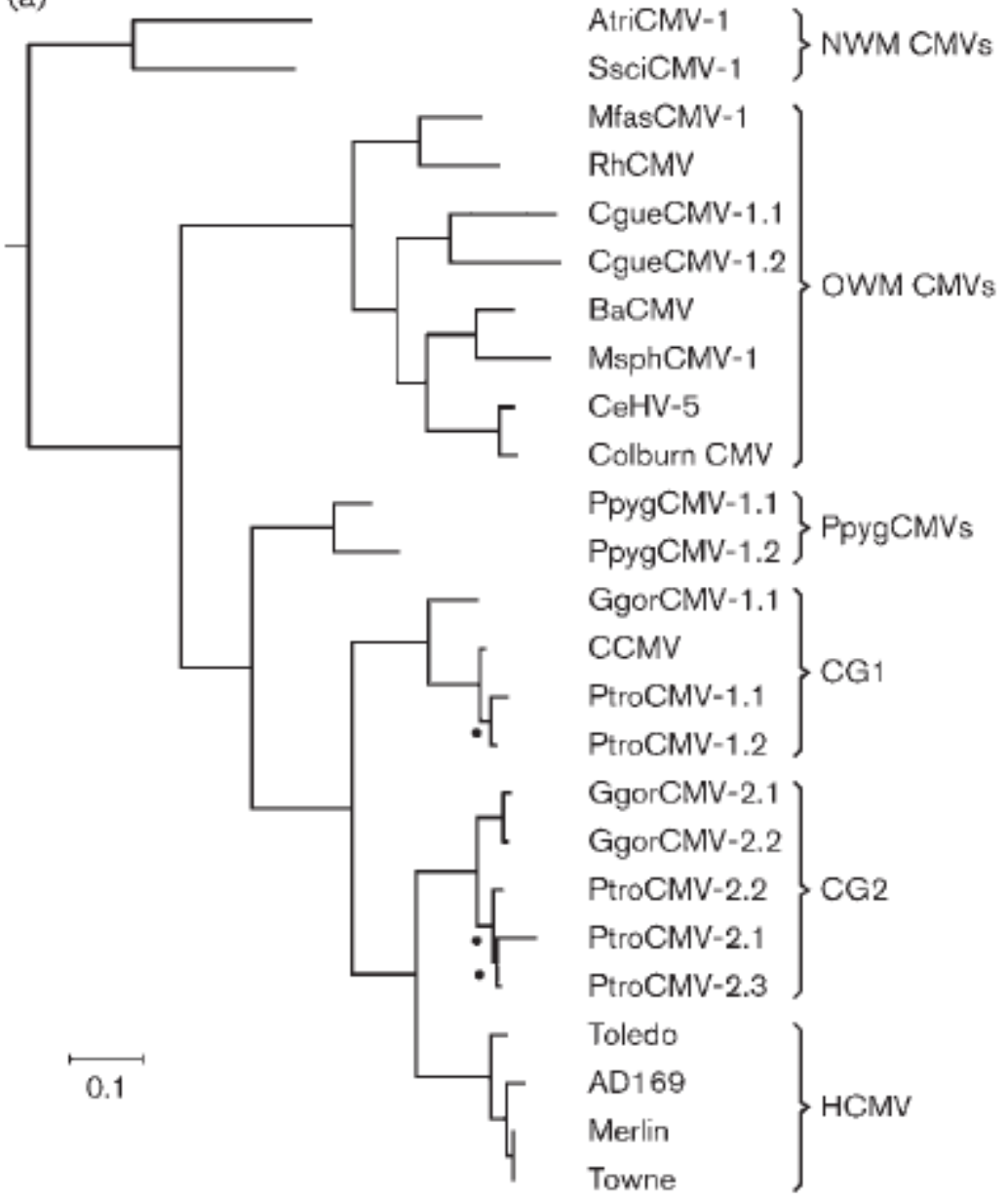

Figure 1 Phylogenetic trees for primate CMVs. (a) Tree based on partial UL55 (gB) sequences. The tree was derived from a 420 aa alignment using BMCMC (MrBayes), with outgroup provided by MCMV, RCMV and TuHV-1 (not shown). All branches have a posterior probability of 1.00 , except for three marked with $\bullet$, whose posterior probabilities are ,0.95. Names for six clades discussed in the text are shown on the right. (b) Tree based on amino acid sequences from genes UL55 and UL56. The tree was derived from a 1127 aa alignment concatenated from UL55 and UL56, as for (a). All branches have a posterior probability of 1.00, except within the HCMV clade. Bars, 0.1 substitutions per site. Note that divergence scales for (a) and (b) differ. (b)

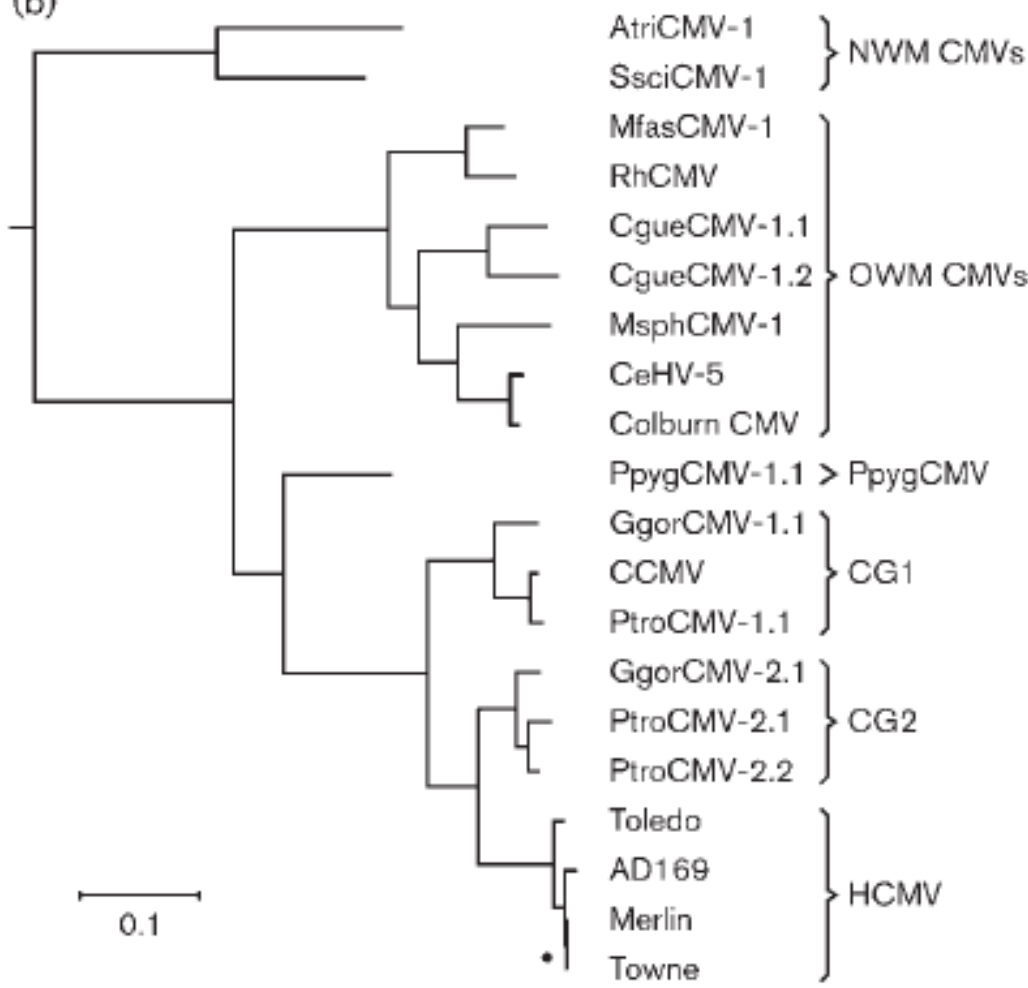


(a)

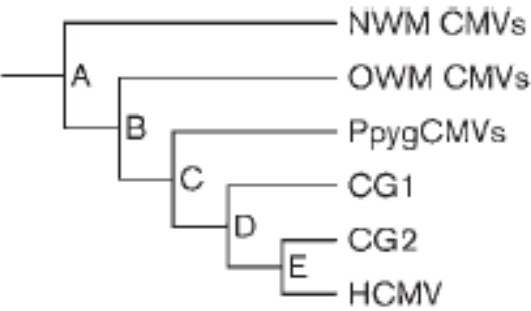

(b)

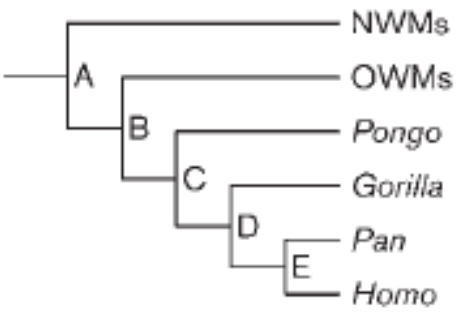

Figure 2 Cladograms for major clades of primate CMVs and host clades. (a) Cladogram for the six primate CMV clades labelled in Fig. 1(a). Nodes are labelled A-E. (b) Cladogram for primate hosts, adapted from Steiper \& Young (2006), showing NWM and OWM clades and individual clades for four hominoid genera, with nodes labelled A-E. 